\title{
Extension of the Poincaré group with half-integer spin generators: hypergravity and beyond
}

\author{
Oscar Fuentealba, ${ }^{a, b}$ Javier Matulich ${ }^{a}$ and Ricardo Troncoso $^{a}$ \\ ${ }^{a}$ Centro de Estudios Cientificos (CECs), \\ Av. Arturo Prat 514, Valdivia, Chile \\ ${ }^{b}$ Departamento de Fúsica, Universidad de Concepción, \\ Casilla 160-C, Concepción, Chile \\ E-mail: fuentealba@cecs.cl, matulich@cecs.cl, troncoso@cecs.cl
}

Abstract: An extension of the Poincaré group with half-integer spin generators is explicitly constructed. We start discussing the case of three spacetime dimensions, and as an application, it is shown that hypergravity can be formulated so as to incorporate this structure as its local gauge symmetry. Since the algebra admits a nontrivial Casimir operator, the theory can be described in terms of gauge fields associated to the extension of the Poincaré group with a Chern-Simons action. The algebra is also shown to admit an infinite-dimensional non-linear extension, that in the case of fermionic spin- $3 / 2$ generators, corresponds to a subset of a contraction of two copies of $\mathrm{WB}_{2}$. Finally, we show how the Poincaré group can be extended with half-integer spin generators for $d \geq 3$ dimensions.

Keywords: Conformal and W Symmetry, Space-Time Symmetries, Gauge-gravity correspondence, Classical Theories of Gravity

ARXIV EPRINT: 1505.06173 


\section{Contents}

1 Introduction 1

$\begin{array}{lll}2 & \text { Fermionic spin-3/2 generators } & 2\end{array}$

2.1 Hypergravity 2

$3 \quad$ Fermionic generators of $\operatorname{spin} s=n+\frac{1}{2} \quad 3$

3.1 Hypergravity in the generic case 4

4 Ending remarks $\quad 6$

\section{Introduction}

Nowadays, we have the good fortune of witnessing the era in which the simplest minimal realistic versions of supersymmetric field theories are about to be either tested or falsified by the LHC. The underlying geometric structure of these kind of theories, as well as most of their widely studied extensions, relies on the super-Poincaré group (see, e.g., [1-3]). According to the Haag-Łopuszański-Sohnius theorem [4], this is a consistent extension of the Poincaré group that includes fermionic generators of spin $1 / 2$. Indeed, in flat spacetimes of dimension greater than three, the addition of fermionic generators of spin $s \geq 3 / 2$ would imply that the irreducible representations necessarily contained higher spin fields, which are known to suffer from inconsistencies (see, e.g., [5-11]). However, in three spacetime dimensions, higher spin fields do not possess local propagating degrees of freedom, and as a consequence, it is possible to describe them consistently [12-18] even on locally flat spacetimes [19-22]. Hence, in the latter context, since no-go theorems about massless higher spin fields can be circumvented, it is natural to look for an extension of the Poincaré group with fermionic half-integer spin generators. Results along these lines have already been explored in [23]. In what follows, we begin with the construction of the searched for extension of the Poincaré group in the case of spin $3 / 2$ generators, that for short, hereafter we dub it the "hyper-Poincaré" group. It is shown that the algebra admits a nontrivial Casimir operator and, as an application, we explain how the hypergravity theory of Aragone and Deser [24] can be formulated so as to incorporate the hyper-Poincaré group as its local gauge symmetry. Concretely, we show how hypergravity can be described in terms of hyperPoincaré-valued gauge fields with a Chern-Simons action. The results are then extended to the case of fermionic generators of spin $n+\frac{1}{2}$, as well as to the minimal coupling of General Relativity with gauge fields of spin $n+\frac{3}{2}$, so that the super-Poincaré group and supergravity are recovered for $n=0$. The hyper-Poincaré algebra is also shown to admit an infinite-dimensional nonlinear extension that contains the $\mathrm{BMS}_{3}$ algebra, which in the case of spin-3/2 generators, reduces to a subset of a suitable contraction of two copies of 
$\mathrm{WB}_{2}$. We conclude explaining how the hyper-Poincaré group is extended to the case of $d \geq 3$ dimensions.

\section{Fermionic spin-3/2 generators}

In three spacetime dimensions, the nonvanishing commutators of the Poincaré algebra can be written as

$$
\left[J_{a}, J_{b}\right]=\varepsilon_{a b c} J^{c}, \quad\left[J_{a}, P_{b}\right]=\varepsilon_{a b c} P^{c} .
$$

The additional fermionic generators are assumed to transform in an irreducible spin- $3 / 2$ representation of the Lorentz group, so that they are described by " $\Gamma$-traceless" vectorspinors that fulfill $Q^{a} \Gamma_{a}=0$, where $\Gamma_{a}$ stand for the Dirac matrices. Their corresponding commutation rules with the Lorentz generators are then given by

$$
\left[J_{a}, Q_{\alpha b}\right]=\frac{1}{2}\left(\Gamma_{a}\right)^{\beta}{ }_{\alpha} Q_{\beta b}+\varepsilon_{a b c} Q_{\alpha}^{c} .
$$

Therefore, requiring consistency of the closure as well as the Jacobi identity, implies that the only remaining nonvanishing (anti-) commutators of the algebra read

$$
\left\{Q_{\alpha}^{a}, Q_{\beta}^{b}\right\}=-\frac{2}{3}\left(C \Gamma^{c}\right)_{\alpha \beta} P_{c} \eta^{a b}+\frac{5}{6} \varepsilon^{a b c} C_{\alpha \beta} P_{c}+\frac{1}{6}\left(C \Gamma^{(a)}\right)_{\alpha \beta} P^{b)},
$$

where $C$ is the charge conjugation matrix. ${ }^{1}$ It is then simple to verify that apart from $I_{1}=P^{a} P_{a}$, the algebra admits another Casimir operator given by

$$
I_{2}=2 J^{a} P_{a}+Q_{\alpha}^{a} C^{\alpha \beta} Q_{\beta a},
$$

which implies the existence of an invariant (anti-) symmetric bilinear form, whose only nonvanishing components are of the form

$$
\left\langle J_{a}, P_{b}\right\rangle=\eta_{a b}, \quad\left\langle Q_{\alpha}^{a}, Q_{\beta}^{b}\right\rangle=\frac{2}{3} C_{\alpha \beta} \eta^{a b}-\frac{1}{3} \varepsilon^{a b c}\left(C \Gamma_{c}\right)_{\alpha \beta} .
$$

It is worth highlighting that the inclusion of the higher spin generators $Q_{\alpha}^{a}$ does not jeopardize the causal structure, since there is no need to enlarge the Lorentz group.

\subsection{Hypergravity}

In order to describe a massless spin- $\frac{5}{2}$ field minimally coupled to General Relativity, let us consider a connection 1-form that takes values in the hyper-Poincaré algebra described above, which reads

$$
A=e^{a} P_{a}+\omega^{a} J_{a}+\psi_{a}^{\alpha} Q_{\alpha}^{a},
$$

\footnotetext{
${ }^{1}$ In our conventions, the Minkowski metric $\eta_{a b}$ is assumed to follow the "mostly plus" convention, and the Levi-Civita symbol fulfills $\varepsilon_{012}=1$. Round brackets stand for symmetrization of the enclosed indices, without the normalization factor, e.g., $X^{(a \mid} Y^{b} Z^{\mid c)}=X^{a} Y^{b} Z^{c}+X^{c} Y^{b} Z^{a}$. It is also useful to keep in mind the Fierz expansion of the product of three Dirac matrices, given by $\Gamma^{a} \Gamma^{b} \Gamma^{c}=\varepsilon^{a b c}+\eta^{a b} \Gamma^{c}+\eta \Gamma^{a}-\eta^{a c} \Gamma^{b}$. Afterwards, the presence of the imaginary unit " $i$ " in the product of real Grassmann variables is because we assume that $\left(\theta_{1} \theta_{2}\right)^{*}=-\theta_{1} \theta_{2}$.
} 
where $e^{a}, \omega^{a}$ and $\psi_{a}^{\alpha}$ stand for the dreibein, the dualized spin connection $\left(\omega^{a}=\frac{1}{2} \varepsilon^{a b c} \omega_{b c}\right)$, and the $\Gamma$-traceless spin- $\frac{5}{2}$ field $\left(\Gamma^{a} \psi_{a}=0\right)$, respectively. The components of the field strength $F=d A+A^{2}$ are then given by

$$
F=R^{a} J_{a}+\tilde{T}^{a} P_{a}+D \psi_{a}^{\alpha} Q_{\alpha}^{a},
$$

where the covariant derivative of the spin- $\frac{5}{2}$ field can be written as $D \psi^{a}=d \psi^{a}+\frac{3}{2} \omega^{b} \Gamma_{b} \psi^{a}-$ $\omega_{b} \Gamma^{a} \psi^{b}$, and $R^{a}=d \omega^{a}+\frac{1}{2} \varepsilon^{a b c} \omega_{b} \omega_{c}$ is the dualized curvature 2-form. The hypercovariant torsion 2 -form then reads

$$
\tilde{T}^{a}:=T^{a}-\frac{3}{4} i \bar{\psi}_{b} \Gamma^{a} \psi^{b},
$$

with $T^{a}=d e^{a}+\varepsilon^{a b c} \omega_{b} e_{c}$, and $\bar{\psi}_{a \alpha}=\psi_{a}^{\beta} C_{\beta \alpha}$ is the Majorana conjugate.

Note that under an infinitesimal gauge transformation $\delta A=d \lambda+[A, \lambda]$, spanned by a hyper-Poincaré-valued zero-form given by $\lambda=\lambda^{a} P_{a}+\sigma^{a} J_{a}+\epsilon_{a}^{\alpha} Q_{\alpha}^{a}$, the components of the gauge field transform according to

$$
\begin{aligned}
\delta e^{a} & =D \lambda^{a}-\varepsilon^{a b c} \sigma_{b} e_{c}+\frac{3}{2} i \bar{\epsilon}_{b} \Gamma^{a} \psi^{b}, \\
\delta \omega^{a} & =D \sigma^{a}, \\
\delta \psi^{a} & =-\frac{3}{2} \sigma^{b} \Gamma_{b} \psi^{a}+\sigma_{b} \Gamma^{a} \psi^{b}+D \epsilon^{a} .
\end{aligned}
$$

The invariant bilinear form (2.5) then allows to construct a Chern-Simons action for the gauge field (2.6), given by

$$
I=\frac{k}{4 \pi} \int\left\langle A d A+\frac{2}{3} A^{3}\right\rangle,
$$

which up to a boundary term, reduces to

$$
I=\frac{k}{4 \pi} \int 2 R^{a} e_{a}+i \bar{\psi}_{a} D \psi^{a} .
$$

It is worth pointing out that, despite the action (2.11) is formally the same as the one considered by Aragone and Deser in [24], it does possess a different local structure. Indeed, note that under local hypersymmetry transformations spanned by $\lambda=\epsilon_{a}^{\alpha} Q_{\alpha}^{a}$, the nonvanishing transformation rule for the spin connection considered in [24], agrees with ours only on-shell. Actually, by construction, as in the case of supergravity [25], here the algebra of the local gauge symmetries (2.9) closes off-shell according to the hyper-Poincaré group, without the need of auxiliary fields.

In the case of negative cosmological constant, it can be seen that hypergravity requires the presence of additional spin-4 fields [26-28].

\section{$3 \quad$ Fermionic generators of $\operatorname{spin} s=n+\frac{1}{2}$}

In this case, the fermionic generators correspond to tensor-spinors $Q_{\alpha}^{a_{1} \ldots a_{n}}$, transforming in an irreducible representation of the Lorentz group, so that they are completely symmetric 
in the vector indices, as well as $\Gamma$-traceless, i.e., $Q^{a_{1} \ldots a_{n}} \Gamma_{a_{1}}=0$. These conditions imply that their anticommutation rules acquire a somehow cumbersome expression, and it is then more convenient to write the hyper-Poincaré algebra in the Maurer-Cartan formalism. The Maurer-Cartan 1-form is given by

$$
\Omega=\rho^{a} P_{a}+\tau^{a} J_{a}+\chi_{a_{1} \ldots a_{n}}^{\alpha} Q_{\alpha}^{a_{1} \ldots a_{n}},
$$

where $\chi_{a_{1} \ldots a_{n}}$ is $\Gamma$-traceless and completely symmetric in the vector indices, which can be seen as a flat connection that fulfills

$$
\begin{aligned}
d \tau^{a} & =-\frac{1}{2} \epsilon^{a b c} \tau_{b} \tau_{c}, \\
d \rho^{a} & =-\epsilon^{a b c} \tau_{b} \rho_{c}+\frac{1}{2}\left(n+\frac{1}{2}\right) i \bar{\chi}_{a_{1} \ldots a_{n}} \Gamma^{a} \chi^{a_{1} \ldots a_{n}}, \\
d \chi^{a_{1} \ldots a_{n}} & =-\left(n+\frac{1}{2}\right) \tau_{b} \Gamma^{b} \chi^{a_{1} \ldots a_{n}}+\tau_{b} \Gamma^{\left(a_{1}\right.} \chi^{\left.a_{2} \ldots a_{n}\right) b} .
\end{aligned}
$$

Note that the Jacobi identity now translates into the consistency of the nilpotence of the exterior derivative $\left(d^{2}=0\right)$, which for the algebra (3.2) is clearly satisfied.

The nontrivial Casimir operator now reads

$$
I_{2}=2 J^{a} P_{a}+Q_{\alpha a_{1} \ldots a_{n}} C^{\alpha \beta} Q_{\beta}^{a_{1} \ldots a_{n}} .
$$

It is also worth pointing out that the super-Poincaré algebra corresponds to the case of $n=0$, while the hyper-Poincaré algebra described above is recovered for $n=1$.

\subsection{Hypergravity in the generic case}

The minimal coupling of General Relativity with a massless fermionic field of spin $s=n+\frac{3}{2}$, described by a completely symmetric $\Gamma$-traceless 1 -form $\psi_{a_{1} \ldots a_{n}}$, can then be formulated in terms of a gauge field for the hyper-Poincaré algebra, which now reads

$$
A=e^{a} P_{a}+\omega^{a} J_{a}+\psi_{a_{1} \ldots a_{n}}^{\alpha} Q_{\alpha}^{a_{1} \ldots a_{n}} .
$$

The components of the curvature 2-form are then given by

$$
F=R^{a} J_{a}+\tilde{T}^{a} P_{a}+D \psi_{a_{1} \ldots a_{n}}^{\alpha} Q_{\alpha}^{a_{1} \ldots a_{n}}
$$

where the covariant derivative of the spin- $\left(n+\frac{3}{2}\right)$ field can be written as

$$
D \psi^{a_{1} \ldots a_{n}}=d \psi^{a_{1} \ldots a_{n}}+\left(n+\frac{1}{2}\right) \omega_{b} \Gamma^{b} \psi^{a_{1} \ldots a_{n}}-\omega_{b} \Gamma^{\left(a_{1}\right.} \psi^{\left.a_{2} \ldots a_{n}\right) b},
$$

and

$$
\tilde{T}^{a}=T^{a}-\frac{1}{2}\left(n+\frac{1}{2}\right) i \bar{\psi}_{a_{1} \ldots a_{n}} \Gamma^{a} \psi^{a_{1} \ldots a_{n}} .
$$

The transformation rules of the fields under local hypersymmetry can then be obtained from a gauge transformation of the connection (3.4) with a fermionic parameter given by 
$\lambda=\epsilon_{a_{1} \ldots a_{n}}^{\alpha} Q_{\alpha}^{a_{1} \ldots a_{n}}$, so that they read

$$
\begin{aligned}
\delta e^{a} & =\left(n+\frac{1}{2}\right) i \bar{\epsilon}_{a_{1} \ldots a_{n}} \Gamma^{a} \psi^{a_{1} \ldots a_{n}}, \\
\delta \omega^{a} & =0 \\
\delta \psi^{a_{1} \ldots a_{n}} & =D \epsilon^{a_{1} \ldots a_{n}} .
\end{aligned}
$$

The Casimir operator (3.3) then implies the existence of an (anti-) symmetric tensor of rank 2 , which once contracted with the wedge product of two curvatures, gives

$$
\begin{aligned}
\left\langle F^{2}\right\rangle & =2 R^{a} \tilde{T}_{a}+i D \bar{\psi}_{a_{1} \ldots a_{n}} D \psi^{a_{1} \ldots a_{n}} \\
& =d\left(2 R^{a} e_{a}+i \bar{\psi}_{a_{1} \ldots a_{n}} D \psi^{a_{1} \ldots a_{n}}\right),
\end{aligned}
$$

being an exact form that is manifestly invariant under the hypersymmetry transformations (3.8). Therefore, as in the case of (super)gravity [29, 30], the action can also be written as a Chern-Simons one (2.10), which up to a boundary term reduces to

$$
I=\frac{k}{4 \pi} \int 2 R^{a} e_{a}+i \bar{\psi}_{a_{1} \ldots a_{n}} D \psi^{a_{1} \ldots a_{n}}
$$

so that the field equations now read $F=0$, with $F$ given by (3.5).

Note that the standard supergravity action in [31-33] is recovered for $n=0$; and as it occurs in the spin- $\frac{5}{2}$ case, the generic theory agrees with the one of Aragone and Deser only on-shell.

We would like to stress that a deeper understanding of the theory cannot be attained unless it is endowed with a consistent set of boundary conditions. In this sense, one of the advantages of formulating hypergravity as a Chern-Simons theory is that the analysis of its asymptotic structure can be directly performed in a canonical form, as in the case of negative cosmological constant [28]. Indeed, in analogy with the case of three-dimensional flat supergravity [34], the mode expansion of the asymptotic symmetry algebra of hypergravity with a spin- $\frac{5}{2}$ fermionic field is defined through the following Poisson brackets [35]

$$
\begin{aligned}
i\left\{\mathcal{J}_{m}, \mathcal{J}_{n}\right\}= & (m-n) \mathcal{J}_{m+n}, \\
i\left\{\mathcal{J}_{m}, \mathcal{P}_{n}\right\}= & (m-n) \mathcal{P}_{m+n}+k m\left(m^{2}-1\right) \delta_{m+n, 0}, \\
i\left\{\mathcal{P}_{m}, \mathcal{P}_{n}\right\}= & 0, \quad i\left\{\mathcal{P}_{m}, \psi_{n}\right\}=0, \\
i\left\{\mathcal{J}_{m}, \psi_{n}\right\}= & \left(\frac{3 m}{2}-n\right) \psi_{m+n}, \\
i\left\{\psi_{m}, \psi_{n}\right\}= & \frac{1}{4}\left(6 m^{2}-8 m n+6 n^{2}-9\right) \mathcal{P}_{m+n}+\frac{9}{4 k} \sum_{q} \mathcal{P}_{m+n-q} \mathcal{P}_{q} \\
& +k\left(m^{2}-\frac{9}{4}\right)\left(m^{2}-\frac{1}{4}\right) \delta_{m+n, 0},
\end{aligned}
$$

which describe a nonlinear hypersymmetric extension of the $\mathrm{BMS}_{3}$ algebra [36-38]. It can also be shown that this algebra corresponds to a subset of a suitable contraction of two copies of the $\mathrm{WB}_{2}$ algebra $[28,39,40]$. 
When fermions fulfill antiperiodic boundary conditions, the modes of the fermionic global charges $\psi_{m}$ are labelled by half-integers, so that the wedge algebra of (3.11) reduces to the one of the hyper-Poincaré group. In fact, dropping the nonlinear terms, and restricting the modes according to $|n|<\Delta$, where $\Delta$ stands for the conformal weight of the generators, the hyper-Poincaré algebra is manifestly recovered provided the modes in (3.11) are identified with the generators $J_{a}, P_{a}, Q_{\alpha a}$, according to

$$
\begin{aligned}
& \mathcal{J}_{-1}=-2 J_{0}, \quad \mathcal{J}_{1}=J_{1}, \quad \mathcal{J}_{0}=J_{2}, \\
& \mathcal{P}_{-1}=-2 P_{0}, \quad \mathcal{P}_{1}=P_{1}, \quad \mathcal{P}_{0}=P_{2}, \\
& \psi_{-\frac{3}{2}}=2^{\frac{5}{4}} \sqrt{3} Q_{+0}, \quad \psi_{-\frac{1}{2}}=2^{\frac{3}{4}} \sqrt{3} Q_{-0}, \\
& \psi_{\frac{1}{2}}=-2^{\frac{1}{4}} \sqrt{3} Q_{+1}, \quad \quad \psi_{\frac{3}{2}}=-2^{-\frac{1}{4}} \sqrt{3} Q_{-1},
\end{aligned}
$$

It is also worth noting that (3.11) can then be regarded as a hypersymmetric extension of the Galilean conformal algebra in two dimensions [41, 42], which is isomorphic to $\mathrm{BMS}_{3}$ and turns out to be relevant in the context of non-relativistic holography.

Another advantage of formulating hypergravity in terms of a Chern-Simons action is that, as in case of supergravity [34, 43], the theory can be readily extended to include parity odd terms in the Lagrangian. This can be explicitly performed by a simple modification of the invariant bilinear form, so that it acquires an additional component given by $\left\langle J_{a}, J_{b}\right\rangle=$ $\mu \eta_{a b}$, followed by a shift in the spin connection of the form $\omega^{a} \rightarrow \omega^{a}+\gamma e^{a}$, so that the constants $\mu, \gamma$ parametrize the new allowed couplings in the action. As a consecuence, when hypergravity is extended in this way, the hyper-BMS $\mathrm{BM}_{3}$ algebra (3.11) acquires an additional nontrivial central extension along its Virasoro subgroup.

\section{Ending remarks}

The hyper-Poincaré group admits a consistent generalization to the case of $d \geq 3$ spacetime dimensions. In the case of fermionic $\Gamma$-traceless spin- $\frac{3}{2}$ generators, the nonvanishing (anti-) commutators of the algebra are given by

$$
\begin{aligned}
{\left[J_{a b}, J_{c d}\right] } & =J_{a d} \eta_{b c}-J_{b d} \eta_{a c}+J_{c a} \eta_{b d}-J_{c b} \eta_{a d}, \\
{\left[J_{a b}, P_{c}\right] } & =P_{a} \eta_{b c}-P_{b} \eta_{a c}, \\
{\left[J_{a b}, Q_{c}^{\alpha}\right] } & =-\frac{1}{2}\left(\Gamma_{a b}\right)^{\alpha}{ }_{\beta} Q_{c}^{\beta}+Q_{a}^{\alpha} \eta_{b c}-Q_{b}^{\alpha} \eta_{a c}, \\
{\left[J_{a b}, \bar{Q}_{\alpha c}\right] } & =\frac{1}{2}\left(\Gamma_{a b}\right)_{\alpha}^{\beta} \bar{Q}_{\beta c}+\bar{Q}_{\alpha a} \eta_{b c}-\bar{Q}_{\alpha b} \eta_{a c}, \\
\left\{Q^{\alpha a}, \bar{Q}_{\beta}^{b}\right\} & =\frac{3(d-2)}{d^{2}} i\left[(d+1)\left(\Gamma^{c}\right)^{\alpha}{ }_{\beta} P_{c} \eta^{a b}-\frac{d+2}{d-2}\left(\Gamma^{a b c}\right)_{\beta}^{\alpha} P_{c}-\left(\Gamma^{(a \mid}\right)_{\beta}^{\alpha} P^{\mid b)}\right],
\end{aligned}
$$

where $\bar{Q}_{a}=Q_{a}^{\dagger} \Gamma^{0}$ stands for the Dirac conjugate.

In the generic case, the spin- $\left(n+\frac{1}{2}\right)$ generators correspond to completely symmetric $\Gamma$ traceless tensor-spinors that fulfill $\Gamma^{a_{1}} Q_{a_{1} \ldots a_{n}}=0$. In order to avoid the intricacies related to the latter condition, as well as with the suitable (anti-) symmetrization of the (anti-) 
commutation rules of the generators, it is better to express the algebra in terms of its Maurer-Cartan form. It is now given by

$$
\Omega=\rho^{a} P_{a}+\frac{1}{2} \tau^{a b} J_{a b}+\bar{\chi}_{\alpha}^{a_{1} \ldots a_{n}} Q_{a_{1} \ldots a_{n}}^{\alpha}-\bar{Q}_{\alpha}^{a_{1} \ldots a_{n}} \chi_{a_{1} \ldots a_{n}}^{\alpha}
$$

where $\chi_{a_{1} \ldots a_{n}}$ is $\Gamma$-traceless and completely symmetric in the vector indices, so that its components fulfill ${ }^{2}$

$$
\begin{aligned}
d \tau^{a b} & =-\tau^{a}{ }_{c} \tau^{c b}, \\
d \rho^{a} & =-\tau^{a}{ }_{b} \rho^{b}+\frac{1}{2}\left(n+\frac{1}{2}\right) i \bar{\chi}_{a_{1} \ldots a_{n}} \Gamma^{a} \chi^{a_{1} \ldots a_{n}}, \\
d \chi^{a_{1} \ldots a_{n}} & =-\frac{1}{4} \tau^{a b} \Gamma_{a b} \chi^{a_{1} \ldots a_{n}}-\tau^{\left(a_{1}\right.}{ }_{b} \chi^{\left.a_{2} \ldots a_{n}\right) b}, \\
d \bar{\chi}^{a_{1} \ldots a_{n}} & =-\frac{1}{4} \bar{\chi}^{a_{1} \ldots a_{n}} \tau^{a b} \Gamma_{a b}-\tau^{\left(a_{1}\right.}{ }_{b} \bar{\chi}^{\left.a_{2} \ldots a_{n}\right) b} .
\end{aligned}
$$

This algebra can be easily written in terms of Majorana spinors when they exist, and it reduces to super-Poincaré for $n=0$.

Note that there was no need to enlarge the Lorentz group in order to accommodate the higher spin generators, so that the additional symmetries do not seem to interfere with the causal structure. Indeed, as in the case of supersymmetry, the quotient of the hyperPoincaré group over the Lorentz subgroup now defines a hyperspace which is an extension of Minkowski spacetime with additional $\Gamma$-traceless tensor-spinor coordinates. However, as anticipated by Haag, Łopuszański and Sohnius, the irreducible representations, which could be obtained from suitable hyperfields, necessarily contain higher spin fields. Nevertheless, it would be worth to explore whether the hyper-Poincaré algebra may manifest itself through theories or models whose fundamental fields do not transform as linear multiplets, as it would be the case of nonlinear realizations, hyper-Poincaré-valued gauge fields, or extended objects.

\section{Acknowledgments}

We thank A. Campoleoni, M. Henneaux, G. Lucena Gómez, A. Pérez, R. Rahman and D. Tempo for helpful discussions and enlightening comments. O.F. and R.T. thank the International Solvay Institutes and the ULB for warm hospitality. O.F. thanks Conicyt for financial support. This research has been partially supported by Fondecyt grants No 1130658, 1121031, 3150448. The Centro de Estudios Científicos (CECs) is funded by the Chilean Government through the Centers of Excellence Base Financing Program of Conicyt.

\footnotetext{
${ }^{2}$ In the case of $d=2$ spacetime dimensions the algebra is consistent. However, the subset spanned by translations and the fermionic generators is an abelian ideal.
} 
Open Access. This article is distributed under the terms of the Creative Commons Attribution License (CC-BY 4.0), which permits any use, distribution and reproduction in any medium, provided the original author(s) and source are credited.

\section{References}

[1] P. Fayet and S. Ferrara, Supersymmetry, Phys. Rept. 32 (1977) 249 [INSPIRE].

[2] M.F. Sohnius, Introducing supersymmetry, Phys. Rept. 128 (1985) 39 [INSPIRE].

[3] P. Van Nieuwenhuizen, Supergravity, Phys. Rept. 68 (1981) 189 [InSPIRE].

[4] R. Haag, J.T. Łopuszański and M. Sohnius, All possible generators of supersymmetries of the S-matrix, Nucl. Phys. B 88 (1975) 257 [INSPIRE].

[5] S. Weinberg, Photons and gravitons in S-matrix theory: derivation of charge conservation and equality of gravitational and inertial mass, Phys. Rev. 135 (1964) B1049 [INSPIRE].

[6] C. Aragone and S. Deser, Consistency problems of hypergravity, Phys. Lett. B 86 (1979) 161 [INSPIRE].

[7] F.A. Berends, J.W. van Holten, P. van Nieuwenhuizen and B. de Wit, On spin $5 / 2$ gauge fields, Phys. Lett. B 83 (1979) 188 [Erratum ibid. B 84 (1979) 529] [INSPIRE].

[8] C. Aragone and S. Deser, Higher spin vierbein gauge fermions and hypergravities, Nucl. Phys. B 170 (1980) 329 [inSPIRE].

[9] S. Weinberg and E. Witten, Limits on massless particles, Phys. Lett. B 96 (1980) 59 [INSPIRE].

[10] M. Porrati, Universal limits on massless high-spin particles, Phys. Rev. D 78 (2008) 065016 [arXiv: 0804.4672] [INSPIRE].

[11] X. Bekaert, N. Boulanger and P.A. Sundell, How higher-spin gravity surpasses the spin-two barrier, Rev. Mod. Phys. 84 (2012) 987 [arXiv:1007.0435] [InSPIRE].

[12] M.P. Blencowe, A consistent interacting massless higher-spin field theory in D=2+1, Class. Quant. Grav. 6 (1989) 443 [inSPIRE].

[13] E. Bergshoeff, M.P. Blencowe and K.S. Stelle, Area preserving diffeomorphisms and higher spin algebra, Commun. Math. Phys. 128 (1990) 213 [INSPIRE].

[14] M.A. Vasiliev, Higher spin gauge theories in four-dimensions, three-dimensions and two-dimensions, Int. J. Mod. Phys. D 5 (1996) 763 [hep-th/9611024] [INSPIRE].

[15] M. Henneaux and S.-J. Rey, Nonlinear $W_{\infty}$ as asymptotic symmetry of three-dimensional higher spin anti-de Sitter gravity, JHEP 12 (2010) 007 [arXiv: 1008.4579] [INSPIRE].

[16] A. Campoleoni, S. Fredenhagen, S. Pfenninger and S. Theisen, Asymptotic symmetries of three-dimensional gravity coupled to higher-spin fields, JHEP 11 (2010) 007 [arXiv: 1008.4744] [INSPIRE].

[17] M. Henneaux, A. Perez, D. Tempo and R. Troncoso, Chemical potentials in three-dimensional higher spin anti-de Sitter gravity, JHEP 12 (2013) 048 [arXiv:1309.4362] [INSPIRE].

[18] C. Bunster, M. Henneaux, A. Perez, D. Tempo and R. Troncoso, Generalized black holes in three-dimensional spacetime, JHEP 05 (2014) 031 [arXiv:1404.3305] [INSPIRE]. 
[19] H. Afshar, A. Bagchi, R. Fareghbal, D. Grumiller and J. Rosseel, Spin-3 gravity in three-dimensional flat space, Phys. Rev. Lett. 111 (2013) 121603 [arXiv:1307.4768] [INSPIRE].

[20] H.A. Gonzalez, J. Matulich, M. Pino and R. Troncoso, Asymptotically flat spacetimes in three-dimensional higher spin gravity, JHEP 09 (2013) 016 [arXiv:1307.5651] [INSPIRE].

[21] M. Gary, D. Grumiller, M. Riegler and J. Rosseel, Flat space (higher spin) gravity with chemical potentials, JHEP 01 (2015) 152 [arXiv:1411.3728] [INSPIRE].

[22] J. Matulich, A. Perez, D. Tempo and R. Troncoso, Higher spin extension of cosmological spacetimes in 3D: asymptotically flat behaviour with chemical potentials and thermodynamics, JHEP 05 (2015) 025 [arXiv:1412.1464] [INSPIRE].

[23] J. Hietarinta, Supersymmetry generators of arbitrary spin, Phys. Rev. D 13 (1976) 838 [INSPIRE].

[24] C. Aragone and S. Deser, Hypersymmetry in D=3 of coupled gravity-massless spin-5/2 system, Class. Quant. Grav. 1 (1984) L9 [INSPIRE].

[25] M. Bañados, R. Troncoso and J. Zanelli, Higher dimensional Chern-Simons supergravity, Phys. Rev. D 54 (1996) 2605 [gr-qc/9601003] [INSPIRE].

[26] B. Chen, J. Long and Y.-N. Wang, Conical defects, black holes and higher spin (super-)symmetry, JHEP 06 (2013) 025 [arXiv:1303.0109] [INSPIRE].

[27] Y.M. Zinoviev, Hypergravity in $A d S_{3}$, Phys. Lett. B 739 (2014) 106 [arXiv:1408.2912] [INSPIRE].

[28] M. Henneaux, A. Perez, D. Tempo and R. Troncoso, Hypersymmetry bounds and three-dimensional higher-spin black holes, arXiv: 1506.01847 [INSPIRE].

[29] A. Achucarro and P.K. Townsend, A Chern-Simons action for three-dimensional anti-de Sitter supergravity theories, Phys. Lett. B 180 (1986) 89 [INSPIRE].

[30] E. Witten, (2+1)-dimensional gravity as an exactly soluble system, Nucl. Phys. B 311 (1988) 46 [inSPIRE].

[31] S. Deser and J.H. Kay, Topologically massive supergravity, Phys. Lett. B 120 (1983) 97 [INSPIRE].

[32] S. Deser, Cosmological topological supergravity, in S.M. Christensen ed., Quantum theory of gravity, CRC Press (1984), pp. 374-381 [INSPIRE].

[33] N. Marcus and J.H. Schwarz, Three-dimensional supergravity theories, Nucl. Phys. B 228 (1983) 145 [inSPIRE].

[34] G. Barnich, L. Donnay, J. Matulich and R. Troncoso, Asymptotic symmetries and dynamics of three-dimensional flat supergravity, JHEP 08 (2014) 071 [arXiv:1407.4275] [INSPIRE].

[35] O. Fuentealba, J. Matulich and R. Troncoso, Asymptotically flat structure of hypergravity in three spacetime dimensions, arXiv:1508.04663 [INSPIRE].

[36] A. Ashtekar, J. Bicak and B.G. Schmidt, Asymptotic structure of symmetry reduced general relativity, Phys. Rev. D 55 (1997) 669 [gr-qc/9608042] [InSPIRE].

[37] G. Barnich and G. Compere, Classical central extension for asymptotic symmetries at null infinity in three spacetime dimensions, Class. Quant. Grav. 24 (2007) F15 [gr-qc/0610130] [INSPIRE]. 
[38] G. Barnich and C. Troessaert, Aspects of the BMS/CFT correspondence, JHEP 05 (2010) 062 [arXiv: 1001.1541] [INSPIRE].

[39] J.M. Figueroa-O'Farrill, S. Schrans and K. Thielemans, On the Casimir algebra of $B_{2}$, Phys. Lett. B 263 (1991) 378 [inSPIRE].

[40] S. Bellucci, S. Krivonos and A.S. Sorin, Linearizing $W_{2,4}$ and $W B_{2}$ algebras, Phys. Lett. B 347 (1995) 260 [hep-th/9411168] [INSPIRE].

[41] A. Bagchi, Correspondence between asymptotically flat spacetimes and nonrelativistic conformal field theories, Phys. Rev. Lett. 105 (2010) 171601 [INSPIRE].

[42] A. Bagchi and R. Gopakumar, Galilean conformal algebras and AdS/CFT, JHEP 07 (2009) 037 [arXiv:0902.1385] [INSPIRE].

[43] A. Giacomini, R. Troncoso and S. Willison, Three-dimensional supergravity reloaded, Class. Quant. Grav. 24 (2007) 2845 [hep-th/0610077] [INSPIRE]. 